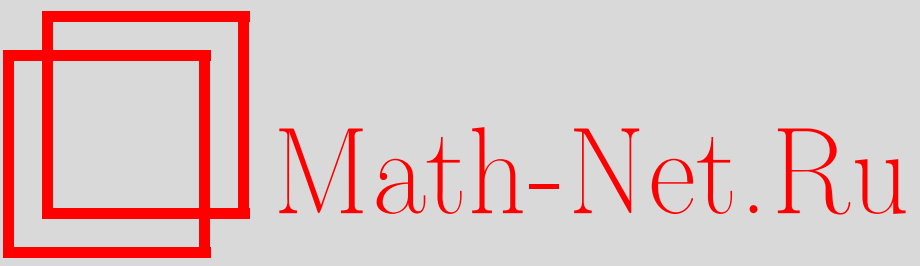

А. А. Серов, Предельное распределение расстояния между случайной булевой функцией и множеством аффинных функций, Теория вероятн. и ее примен., 2010, том 55, выпуск 4, 791-795

DOI: https://doi.org/10.4213/tvp4283

Использование Общероссийского математического портала Math-Net.Ru подразумевает, что вы прочитали и согласны с пользовательским соглашением

http://www . mathnet.ru/rus/agreement

Параметры загрузки:

IP : 54.209 .52 .79

26 апреля 2023 г., 03:37:44 
(c) 2010 г.

СЕРОВ А. А.*

\section{ПРЕДЕЛЬНОЕ РАСПРЕДЕЛЕНИЕ РАССТОЯНИЯ МЕЖДУ СЛУЧАЙНОЙ БУЛЕВОЙ ФУНКЦИЕЙ И МНОЖЕСТВОМ АФФИННЫХ ФУНКЦИЙ ${ }^{1)}$}

Доказана предельная теорема для расстояния Хемминга от случайной равновероятной булевой функции от $n$ переменных до множества аффинных булевых функций от тех же переменных. Проведено сравнение с аналогичной теоремой, доказанной Б. В. Рязановым для расстояния до множества линейных булевых функций.

Ключевые слова и фразы: случайные булевы функции, аффинные булевы функции, расстояние Хемминга, предельные распределения.

1. Введение. Пусть $\mathbf{F}_{2}$ - поле из двух элементов. Для произвольного натурального числа $n$ будем обозначать через $V_{n}$ пространство $n$-мерных векторов с компонентами из $\mathbf{F}_{2}$. Между множеством $\mathbf{F}_{2}^{V_{n}}=\left\{f: V_{n} \rightarrow \mathbf{F}_{2}\right\}$ всех булевых функций от $n$ переменных и пространством $V_{2^{n}}$ можно установить взаимно однозначное соответствие, отождествляя функцию $f \in \mathbf{F}_{2}^{V_{n}}$ с вектором $\left\{f(x) \mid x \in V_{n}\right\}$.

Расстояние Хэмминга $\rho(f, g)$ между булевыми функциями $f, g \in \mathbf{F}_{2}^{V_{n}}$ определяется как число значений аргумента $x \in V_{n}$, при которых $f(x) \neq g(x)$. Для произвольного множества булевых функций $A \subset \mathbf{F}_{2}^{V_{n}}$ и функции $f \in \mathbf{F}_{2}^{V_{n}}$ обозначим через $\rho(f, A)=\min _{g \in A} \rho(f, g)$ расстояние Хэмминга от $f$ до ближайшей к ней функции из множества $A$.

В множестве $\mathbf{F}_{2}^{V_{n}}$ всех булевых функций естественно выделяются класс линейных функций

$$
\mathbf{L}_{n}=\left\{f \in \mathbf{F}_{2}^{V_{n}}: f\left(x_{1}, \ldots, x_{n}\right)=a_{1} x_{1} \oplus \cdots \oplus a_{n} x_{n}, a_{1}, \ldots, a_{n} \in \mathbf{F}_{2}\right\}
$$

и класс аффинных функций

$$
\mathbf{A}_{n}=\left\{f \in \mathbf{F}_{2}^{V_{n}}: f\left(x_{1}, \ldots, x_{n}\right)=a_{0} \oplus a_{1} x_{1} \oplus \cdots \oplus a_{n} x_{n}, a_{0}, \ldots, a_{n} \in \mathbf{F}_{2}\right\},
$$

где $\oplus$ - сложение в $\mathbf{F}_{2}$. Множество $\mathbf{L}_{n}$ состоит из $\left|\mathbf{L}_{n}\right|=2^{n}$ элементов, множество $\mathbf{A}_{n}-$ из $\left|\mathbf{A}_{n}\right|=2^{n+1}$ элементов.

Пусть $f \in \mathbf{F}_{2}^{V_{n}}$ - случайная булева функция, имеющая равномерное распределение на $\mathbf{F}_{2}^{V_{n}}$. При этом условии в [5] было доказано, что

$$
\lim _{n \rightarrow \infty} \mathbf{P}\left\{\frac{\rho\left(f, \mathbf{L}_{n}\right)-c_{n}}{d_{n}}<x\right\}=1-e^{-e^{x}} \quad \forall x \in \mathbf{R},
$$

где

$$
c_{n}=2^{n-1}-2^{(n-1) / 2} \sqrt{\ln 2}\left(\sqrt{n}-\frac{\ln \ln 2^{n}+\ln 4 \pi}{4 \sqrt{n} \ln 2}\right), \quad d_{n}=\frac{2^{(n-1) / 2}}{2 \sqrt{n \ln 2}} .
$$

Основным результатом настоящей статьи является аналогичная предельная теорема для случайной величины $\rho_{n}=\rho\left(f, \mathbf{A}_{n}\right)$.

* Математический институт им. В.А.Стеклова РАН, ул. Губкина, 8, 119991 Москва, ГСП-1, Россия; e-mail: serov1984@mail.ru

1) Работа выполнена при поддержке РФФИ (грант № 08-01-00078). 
Теорема 1. Для любого фиксированного $x \in \mathbf{R}$

$$
\lim _{n \rightarrow \infty} \mathbf{P}\left\{\frac{\rho\left(f, \mathbf{A}_{n}\right)-a_{n}}{b_{n}} \leqslant x\right\}=1-e^{-e^{x}},
$$

где

$$
a_{n}=2^{n-1}-2^{(n-1) / 2} \sqrt{\ln 2}\left(\sqrt{n+1}-\frac{\ln \ln 2^{n+1}+\ln 4 \pi}{4 \sqrt{n+1} \ln 2}\right), \quad b_{n}=\frac{2^{(n-1) / 2}}{2 \sqrt{(n+1) \ln 2}} .
$$

3 а м е ч а н и е. Так как при $n \rightarrow \infty$

$$
\begin{aligned}
c_{n}-a_{n} & =2^{(n-1) / 2} \sqrt{\ln 2}\left(\frac{1}{2 \sqrt{n}}+O\left(\frac{\ln n}{n^{3 / 2}}\right)\right)=\left(1+O\left(\frac{\ln n}{n}\right)\right) d_{n} \ln 2, \\
\frac{d_{n}}{b_{n}} & =\sqrt{\frac{n+1}{n}}=1+O\left(\frac{1}{n}\right),
\end{aligned}
$$

TO

$$
\lim _{n \rightarrow \infty} \mathbf{P}\left\{\frac{\rho\left(f, \mathbf{A}_{n}\right)-c_{n}}{d_{n}} \leqslant x-\ln 2\right\}=1-e^{-e^{x}},
$$

т.е. типичные значения расстояния от случайной равновероятной булевой функции до множества аффинных функций асимптотически на $d_{n} \ln 2=2^{(n-1) / 2} \sqrt{\ln 2 /(4 n)}$ меньше типичных расстояний до множества линейных функций, и это различие имеет тот же порядок, что и длина интервала, содержащего основную массу значений $\rho\left(f, \mathbf{A}_{n}\right)$ или $\rho\left(f, \mathbf{L}_{n}\right)$.

Теорема 1, как и результат Б. В. Рязанова [5], относятся к «центральной» части распределений $\rho\left(f, \mathbf{A}_{n}\right)$ и $\rho\left(f, \mathbf{L}_{n}\right)$. В [7] доказаны неравенства, показывающие, что в области «больших уклонений» (когда $x \rightarrow-\infty$ при $n \rightarrow \infty$ ) правые части формул (1) и (2) не являются правильными асимптотиками вероятностей в их левых частях.

Мы получим теорему 1 как следствие более общей теоремы 2 , в доказательстве которой используются идеи и результаты работ Б. В. Рязанова и С. И. Чечёты [2], [5].

Будем через $\mathbf{I}\{S\}$ обозначать индикатор события $S$.

Введем случайные величины

$$
\begin{aligned}
\xi_{l}(x) & =\mathbf{I}\{f(x)=l(x)\}, \quad l \in \mathbf{A}_{n}, \quad x \in V_{n}, \\
\xi_{l} & =\sum_{x \in V_{n}} \xi_{l}(x)
\end{aligned}
$$

пусть

$$
\xi_{[1]} \geqslant \xi_{[2]} \geqslant \cdots \geqslant \xi_{[k]} \geqslant \cdots \geqslant \xi_{\left[2^{n+1}\right]}
$$

- вариационный ряд построенный по величинам $\xi_{l}, l \in \mathbf{A}_{n}$.

Если случайная функция $f$ имеет равномерное распределение на $\mathbf{F}_{2}^{V_{n}}$, то для любой неслучайной функции $g \in \mathbf{F}_{2}^{V_{n}}$ и для каждого $x \in V_{n}$

$$
\mathbf{P}\left\{\xi_{g}(x)=0\right\}=\mathbf{P}\left\{\xi_{g}(x)=1\right\}=\frac{1}{2},
$$

и индикаторы $\xi_{g}(x), x \in V_{n}$, независимы.

Теорема 2. Пусть $0<\lambda<\infty, c=2^{n-1}+2^{n / 2-1} \tilde{c} u n, \tilde{c} \rightarrow \infty$ mak, ито

$$
\tilde{c}=\left(2 \ln 2^{n+1}-\ln \ln 2^{n+1}-\ln 4 \pi \lambda^{2}+o(1)\right)^{1 / 2} .
$$

Тогда для любого фиксированного $k=1,2, \ldots$

$$
\lim _{n \rightarrow \infty} \mathbf{P}\left\{\xi_{[k]}<c\right\}=e^{-\lambda} \sum_{j=0}^{k-1} \frac{\lambda^{j}}{j !} .
$$


Д о к а з а т е л ь с т в о т е о р е м ы 2. Для любого действительного $c$ введем случайные величины

$$
\eta_{l}=\eta_{l}(c)=\mathbf{I}\left\{\xi_{l} \geqslant c\right\}, \quad \eta=\eta(c)=\sum_{l \in \mathbf{A}_{n}} \eta_{l},
$$

понятно, что $\mathbf{I}\left\{\xi_{l} \geqslant c\right\}=\mathbf{I}\left\{\rho(l, f) \leqslant 2^{n}-c\right\}$.

Очевидное тождество

$$
\mathbf{P}\left\{\xi_{[k]}<c\right\}=\mathbf{P}\{\eta<k\}
$$

позволяет свести задачу о распределении членов вариационного ряда (4) к задаче о распределении целочисленной случайной величины $\eta$, представленной в виде суммы зависимых индикаторов.

Пусть $l_{1}, \ldots, l_{m}$ - различные аффинные функции, а $G_{m}-$ множество всех наборов из $m$ попарно различных аффинных функций. Обозначим

$$
p(\vec{l})=\mathbf{P}\left\{\xi_{l_{i}} \geqslant c, i=1, \ldots, m\right\}, \quad \vec{l}=\left(l_{1}, \ldots, l_{m}\right) \in G_{m} .
$$

Согласно теореме Б.А. Севастьянова [6], для доказательства теоремы 2 достаточно установить, что вероятности $p(\vec{l})$ при $n \rightarrow \infty$ удовлетворяют условиям

$$
\max _{l_{1} \in \mathbf{A}_{n}} p\left(l_{1}\right) \rightarrow 0, \quad \lim _{n \rightarrow \infty} \sum_{l_{1} \in \mathbf{A}_{n}} p\left(l_{1}\right)=\lambda,
$$

и существуют такие исключительные множества $I_{m}(n) \subset G_{m}, n, m \geqslant 2$, наборов $\vec{l}$, что

и

$$
\lim _{n \rightarrow \infty} \sum_{\vec{l} \in I_{m}(n)} p(\vec{l})=\lim _{n \rightarrow \infty} \sum_{\vec{l} \in I_{m}(n)} p\left(l_{1}\right) \cdots p\left(l_{m}\right)=0
$$

$$
\lim _{n \rightarrow \infty} \max _{\vec{l} \in G_{m} \backslash I_{m}(n)}\left|\frac{p(\vec{l})}{p\left(l_{1}\right) \cdots p\left(l_{m}\right)}-1\right|=0 .
$$

Из (3) и из равномерности распределения $f$ на $\mathbf{F}_{2}^{V_{n}}$ следует, что случайная величина $\xi_{l}$ представляет собой сумму $2^{n}$ независимых одинаково распределённых индикаторов $\xi_{l}(x), x \in V_{n}$. Поэтому

$$
\mathbf{M} \xi_{l}=\sum_{x \in V_{n}} \mathbf{M} \xi_{l}(x)=2^{n-1}, \quad \mathbf{D} \xi_{l}=2^{n} \mathbf{D} \xi_{l}(x)=2^{n-2}
$$

Центрирование и нормирование $\xi_{l}$ даёт случайную величину $\tilde{\xi}_{l}=\left(\xi_{l}-\mathbf{M} \xi_{l}\right) / \sqrt{\mathbf{D} \xi_{l}}$, представимую в виде

и

$$
\tilde{\xi}_{l}=\frac{2^{n}-2 \sum_{x \in V_{n}}(f(x) \oplus l(x))}{2^{n / 2}}=2^{-n / 2} \sum_{x \in V_{n}}(-1)^{f(x) \oplus l(x)}
$$

$$
p(\vec{l})=\mathbf{P}\left\{\tilde{\xi}_{l_{i}} \geqslant \tilde{c}, i=1, \ldots, m\right\}, \quad \tilde{c}=\frac{c-2^{n-1}}{2^{n / 2-1}} .
$$

Из (5) видно, что $\tilde{c}=o\left(2^{n / 6}\right)$, поэтому, используя теорему о больших отклонениях $[3$, с. 622$]$ и асимптотику хвоста функции стандартного нормального распределения $\Phi(x)$, находим, что

$$
\mathbf{P}\left\{\tilde{\xi}_{l} \geqslant \tilde{c}\right\} \sim 1-\Phi(\tilde{c}) \sim \frac{e^{-\tilde{c}^{2} / 2}}{\sqrt{2 \pi} \tilde{c}} \quad \text { при } n \rightarrow \infty,
$$

откуда следует первая часть условия (8).

Подставляя выражение для $\tilde{c}$ в правую часть (12), получаем

$$
\frac{e^{-\tilde{c}^{2} / 2}}{\sqrt{2 \pi} \tilde{c}} \sim \frac{\sqrt{4 \pi \lambda^{2} \ln 2^{n+1}}}{2^{n+1} \sqrt{4 \pi\left(1-\left(\ln \ln 2^{n+1}+\ln 4 \pi \lambda^{2}\right) /\left(2 \ln 2^{n+1}\right)\right) \ln 2^{n+1}}} \sim \frac{\lambda}{2^{n+1}} \quad \text { при } n \rightarrow \infty ;
$$


отсюда следует вторая часть (8).

Для любых $n, m \geqslant 2$ и попарно различных аффинных функций $l_{1}, \ldots, l_{m}$ обозначим

$$
I_{m}(n)=\bigcup_{1 \leqslant i<j \leqslant m}\left\{\left(l_{1}, \ldots, l_{m}\right) \in G_{m}: l_{i} \oplus l_{j} \equiv 1\right\} .
$$

Таким образом, если $\vec{l} \in G_{m} \backslash I_{m}(n)$, то для любых $1 \leqslant i<j \leqslant m$

$$
\rho\left(l_{i}, l_{j}\right)=2^{n-1} .
$$

Оценим число элементов в множестве $I_{m}(n)$ :

$$
\begin{aligned}
\left|I_{m}(n)\right| & =C_{m}^{2}\left|\left\{\vec{l} \in G_{m}: l_{m-1} \oplus l_{m}=1\right\}\right| \\
& =C_{m}^{2}\left|\bigcup_{l_{m} \in \mathbf{A}_{n}}\left\{\vec{l} \in G_{m-2}: l_{1}, \ldots, l_{m-2} \notin\left\{l_{m}, l_{m} \oplus 1\right\}\right\}\right| \leqslant C_{m}^{2} 2^{n+1}\left(2^{n+1}-2\right)^{[m-2]},
\end{aligned}
$$

где $x^{[k]}=x(x-1) \cdots(x-k+1)$. Отсюда, из (12) и того, что $\tilde{c} \rightarrow \infty$ при $n \rightarrow \infty$, следует, что при любом $m \geqslant 2$

$$
\sum_{\vec{l} \in I_{m}(n)} p\left(l_{1}\right) \cdots p\left(l_{m}\right) \leqslant \frac{m(m-1)}{2}\left(2^{n+1} \Phi(-\tilde{c})\right)^{m-1} \Phi(-\tilde{c})=o(1), \quad n \rightarrow \infty
$$

т.е. выполняется второе равенство в (9).

Первая часть условия (9) выполняется, так как если $l_{1}, l_{2} \in \mathbf{A}_{n}$ и $l_{1} \oplus l_{2} \equiv 1$, то $\tilde{\xi}_{l_{1}}=-\tilde{\xi}_{l_{2}}$ и события $\left\{\tilde{\xi}_{l_{1}} \geqslant \tilde{c}\right\},\left\{\tilde{\xi}_{l_{2}} \geqslant \tilde{c}\right\}$ несовместны при $\tilde{c}>0$.

Согласно (12) условие (10) принимает вид:

$$
p(\vec{l})=(1+o(1)) \Phi^{m}(-\tilde{c}), \quad n \rightarrow \infty,
$$

равномерно по $\vec{l} \in G_{m} \backslash I_{m}(n)$.

Пусть $\vec{l} \in G_{m} \backslash I_{m}(n)$. Случайный вектор $\tilde{\xi}(\vec{l})=\left(\tilde{\xi}_{l_{1}}, \ldots, \tilde{\xi}_{l_{m}}\right)$ представляет собой центрированную сумму $2^{n}$ независимых случайных векторов $\left(2^{-n / 2} \xi_{l_{1}}(x), \ldots, 2^{-n / 2} \xi_{l_{m}}(x)\right), x \in V_{n}$. Обозначим через $M=\left(m_{i j}\right)$ ковариационную матрицу вектора $\tilde{\xi}(\vec{l})$. Из (11) находим, что

$$
m_{i j}=\mathbf{M} \tilde{\xi}_{l_{i}} \tilde{\xi}_{l_{j}}=\frac{1}{2^{n}} \mathbf{M} \sum_{x \in V_{n}} \sum_{y \in V_{n}}(-1)^{f(x) \oplus l_{i}(x) \oplus f(y) \oplus l_{j}(y)} .
$$

Математические ожидания отдельных слагаемых из последней суммы, вследствие равновероятности $f$ и выбора $\vec{l}$, выглядят так:

$$
\begin{aligned}
& \mathbf{M}(-1)^{f(x) \oplus l_{i}(x) \oplus f(y) \oplus l_{i}(y)}=\left\{\begin{array}{ll}
1, & x=y, \\
0, & x \neq y,
\end{array} \text { если } i=j ;\right. \\
& \mathbf{M}(-1)^{f(x) \oplus l_{i}(x) \oplus f(y) \oplus l_{j}(y)}=\left\{\begin{array}{ll}
(-1)^{l_{i}(x) \oplus l_{j}(x)}, & x=y, \\
0, & x \neq y .
\end{array} \text { если } i \neq j .\right.
\end{aligned}
$$

Следовательно,

$$
m_{i i}=\frac{1}{2^{n}} \sum_{x \in V_{n}} 1=1
$$

а если $i \neq j$, то

$$
m_{i j}=\frac{1}{2^{n}}\left(\sum_{x \in V_{n}}(-1)^{l_{i}(x) \oplus l_{j}(x)}+\sum_{x \in V_{n}} \sum_{y \in V_{n} \backslash\{x\}} \mathbf{M}(-1)^{f(x) \oplus l_{i}(x) \oplus f(y) \oplus l_{j}(y)}\right)=0,
$$

так как в силу условия $\vec{l} \in G_{m} \backslash I_{m}(n)$ функция $l_{i}(x) \oplus l_{j}(x)$ линейна и отлична от константы. 
Тогда справедливость условия (14) вытекает из следствия 1 теоремы 3 работы [2] об асимптотике вероятности попадания суммы независимых случайных $m$-мерных векторов специального вида в область, получающуюся при сдвиге «положительного октанта» в $\mathbf{R}^{m}$ на вектор, все компоненты которого стремятся к $+\infty$.

Таким образом, доказано, что выполняются все условия теоремы Б. А. Севастьянова. Значит, распределение $\eta$ сходится к распределению Пуассона, что эквивалентно утверждению теоремы 2.

Д ок аз а т е ль с т в о т е о р е м ы 1 . Пусть $x \in \mathbf{R}-$ произвольное фиксированное число. Положим

$$
\lambda=e^{x}, \quad c=2^{n-1}+2^{n / 2-1} \tilde{c} \quad \text { и } \quad \tilde{c}=\left(2 \ln 2^{n+1}-\ln \ln 2^{n+1}-\ln 4 \pi \lambda^{2}\right)^{1 / 2} .
$$

Тогда при $n \rightarrow \infty$ выполняются условия теоремы 2 , откуда следует, что

$$
\lim _{n \rightarrow \infty} \mathbf{P}\{\eta(c)=0\}=\lim _{n \rightarrow \infty} \mathbf{P}\left\{\rho\left(f, \mathbf{A}_{n}\right)>2^{n}-c\right\}=e^{-e^{x}} .
$$

Утверждение теоремы 1 состоит в том, что

$$
\lim _{n \rightarrow \infty} \mathbf{P}\left\{\frac{\rho\left(f, \mathbf{A}_{n}\right)-a_{n}}{b_{n}} \leqslant x\right\}=1-e^{-e^{x}} .
$$

Из (15) следует, что доказательство теоремы 1 сводится к проверке соотношения

$$
\frac{2^{n}-c-a_{n}}{b_{n}} \underset{n \rightarrow \infty}{\longrightarrow} x
$$

По определению $a_{n}$ и $b_{n}$

$$
\frac{2^{n}-c-a_{n}}{b_{n}}=\frac{2^{(n-1) / 2} \sqrt{\ln 2^{n+1}}\left(1-\left(\ln \ln 2^{n+1}+\ln 4 \pi\right) /\left(4 \ln 2^{n+1}\right)\right)-2^{(n-1) / 2}(\tilde{c} / \sqrt{2})}{\left(2^{(n-1) / 2}\right) /\left(2 \sqrt{\ln 2^{n+1}}\right)} ;
$$

отсюда и из равенства

$$
\tilde{c}=\sqrt{2 \ln 2^{n+1}}\left(1-\frac{\ln \ln 2^{n+1}+\ln 4 \pi+2 x}{4 \ln 2^{n+1}}+O\left(\frac{\ln ^{2} n}{n^{2}}\right)\right)
$$

следует (16). Теорема 1 доказана.

Автор признателен А. М. Зубкову за постановку задачи и внимание.

\section{СПИСОК ЛИТЕРАТУРЫ}

1. Логачев О.А., Сальников А.А., Ященко В. В. Булевы функции в теории кодирования и криптологии. М.: МЦНМО, 2004.

2. Рязанов Б. В., Чечёта С. И. О приближении случайной булевой функции множеством квадратичных форм. - Дискретн. матем., 1995, т. 7, № 3, с. 129-145.

3. Феллер В. Введение в теорию вероятностей и ее приложения, т. 2. М.: Мир, 1984.

4. Чечёта С.И. О предельном распределении расстояния между случайным вектором и некоторыми двоичными кодами. - Проблемы передачи информации, 1995, т. 31, № 1, c. 90-98.

5. Ryasanov B. V. Probabilistic methods in the theory of approximation of discrete functions. - Probab. Meth. Discr. Math., Proc. 3rd Int. Petrozavodsk Conf. TVP/VSP, Moscow, 1993, p. 403-412.

6. Колчин В.Ф., Севастьянов Б. А., Чистяков В. П. Случайные размещения. М.: Наука, 1976.

7. Зубков А.М., Серов А.А. Оценки числа булевых функций, имеющих аффинных приближения заданной точности. - Дискретн. матем., 2010, т. 22, № 4, с. 3-19.

Поступила в редакцию 20.V.2010 